\title{
Bogidiella martini, un nouvel Amphipode souterrain de l'Ile Saint-Martin (Antilles) et la zoogéographie des Bogidiellidae
}

par

Jan H. STOCK*

Bogidiella martini, a new hypogean Amphipod from the island of Saint-Martin (West Indies) and the zoogeography of the Bogidiellidae.

\begin{abstract}
Summary
Both sexes of a new species of Bogidiella, B. martini, are described. The new species, with a very pronounced sexual dimorphism, has been discovered in two wells in the island of St.-Martin (French part), one of the Lesser Antilles. Another member of Bogidiella has been recorded from the island of Curaçao, but the specimens were damaged too much to allow proper description.

The Bogidiellidae (5 genera, 26 named species, several unnamed species) are present in the sea as well as in inland waters. The family has a wide distribution, exceeding the boundaries of the former Tethys Sea. Probably, they represent a very old stock that had acquired already a great part of its present-day distribution before the fragmentation of the primordial continent of Pangaea during the Mesozoic.
\end{abstract}

Saint-Martin est une île relativement petite $(13 \times 15 \mathrm{~km})$ dans la chaîne des Antilles Calcaires, dont la partie Nord, française, appartient administrativement à la Guadeloupe, tandis que la moitié Sud fait partie des Antilles Néerlandaises. Malgré sa petite taille, l'île possède une faune aquatique hypogée assez remarquable, avec un Mysidacé (Stygiomysis holthuisi (Gordon)), un Amphipode Hadziidé (Metaniphargus palpator Stock), un Isopode Anthuridé (Cyathura sp.) et un Gastéropode (Caecum sp.). Un nouveau membre de cette faune sera décrit dans la présente note. Il s'agit d'un Amphipode nouveau appartenant à la famille des Bogidiellidae.

Je tiens à remercier la Fondation néerlandaise pour le Développement de la Recherche tropicale ("WOTRO", La Haye) et la Société de la Recherche scientifique tropicale ("Treub Maatschappij", Utrecht) pour leur support financier des travaux sur le terrain en 1974 et 1976.

\footnotetext{
* Institut de Zoologie taxonomique (Zoölogisch Museum), Université d'Amsterdam, Pays-Bas.
} 
Bogidiella martini n. sp. (figs. 1-30)

\begin{abstract}
Matériel examiné. - Tous de lîle St.-Martin, partie française:
- Station $74 / 9$, puits dans une vallée à sec au pied de la pente Est du Mont Vernon ( $18^{\circ} 05^{\prime} 07^{\prime \prime} \mathrm{N}$ $63^{\circ} 01^{\prime} 45^{\prime \prime} \mathrm{W}$ ); altitude $10-20 \mathrm{~m}$; température $25^{\circ} 3 \mathrm{C}$; chlorinité $780 \mathrm{mg} / \mathrm{l}$; I févr. 1974; 1 ô (holotype).

type).

- Station 76/41, puits dans la Ravine du Colombier, pas loin de l'Anse des Pères ( $18^{\circ} 05^{\prime} 03^{\prime \prime} \mathrm{N}$ $63^{\circ} 04^{\prime} 46^{\prime \prime} \mathrm{W}$ ); altitude $9 \mathrm{~m}$; chlorinité $500 \mathrm{mg} / \mathrm{l} ; 16$ juin 1976; 1 q à oostégites (paratype).
\end{abstract}

Description. - Longueur du mâle entre 2 et $3 \mathrm{~mm}$, de la femelle à oostégites $11 / 2 \mathrm{~mm}$. Aveugle. Tous les individus sont amputés de leurs péréiopodes 7 . Le sinus de l'antenne inférieure sur la tête est à peine prononcé (fig. 1).

Antenne supérieure (fig. 2) très courte, moins d'un tiers de la longueur du corps. Les articles 1 à 3 du pédoncule ont une longueur de 286, 223 et $133 \mu \mathrm{m}$ (basé sur l'holotype). Trois épines ornent le bord ventral du premier article. Le flagelle accessoire est biarticulé, sa longueur ( $\hat{\sigma}$ ) est de $148 \mu \mathrm{m}$; il est légèrement plus court $(\hat{\delta})$ ou aussi long $(q)$ que les trois articles proximaux du flagelle principal (fig. 3). Le flagelle principal compte 8 ou 9 articles; chaque article porte un aesthète distal, parfois (p. ex. sur l'article 3 du mâle illustré en fig. 3) aussi un aesthète subbasal.

L'antenne inférieure (fig. 4) a pratiquement la même longueur que l'A 1. Les deux derniers articles du pédoncule sont allongés, le pénultième (ô ) est de $333 \mu \mathrm{m}$, le dernier est de $280 \mu \mathrm{m}$; ils sont ornés, surtout à leur angle ventrodistal, de nombreuses soies simples. Le fouet possède 5 articles.

La mandibule porte un palpe triarticulé (fig. 7), dont l'article basal est relativement court, tandis que les articles 2 et 3 sont subégaux et grêles; l'article 2 porte une seule soie assez longue; l'article 3 porte 2 soies subdistales et 2 soies distales. Les parties masticatoires des mandibules droite et gauche sont asymétriques: A gauche, il existe une lacinia mobilis à base étroite, distalement dilatée et ornée d'une rangée de dents (fig. 6); à droite, une lacinia mobilis fait défaut (fig. 5). La pars incisiva gauche est armée d'une série de dents dont deux fortement développées; la pars incisiva droite comporte deux rangées parallèles de dents irrégulières. La pars molaris porte quelques petites dents et une courte soie; elle est identique à gauche et à droite. Entre la pars molaris et la pars incisiva s'insèrent une (gauche) ou trois (droite) épines foliacées et une rangée de cils.

La première maxille (fig. 8) se compose d'un lobe interne quasi-circulaire portant 2 soies distales, d'un lobe externe avec 7 fortes épines, et d'un palpe biarticulé avec 2 soies à son sommet. Les épines du lobe externe (fig. 9) sont ornées de façon différente: les 4 épines les plus courtes sont bifides, les 3 épines les plus longues sont pluridenticulées dans leur région médiane.

La deuxième maxille (fig. 10) comporte 2 lobes, dont l'interne porte 3 paires de soies plumeuses et 2 soies simples, tandis que l'externe porte 4 paires de soies simples de longueur plus importante.

Le maxillipède (fig. 25) est conforme au type habituel pour le genre. Les articles du palpe sont relativement allongés, le 3ème article porte une rangée 


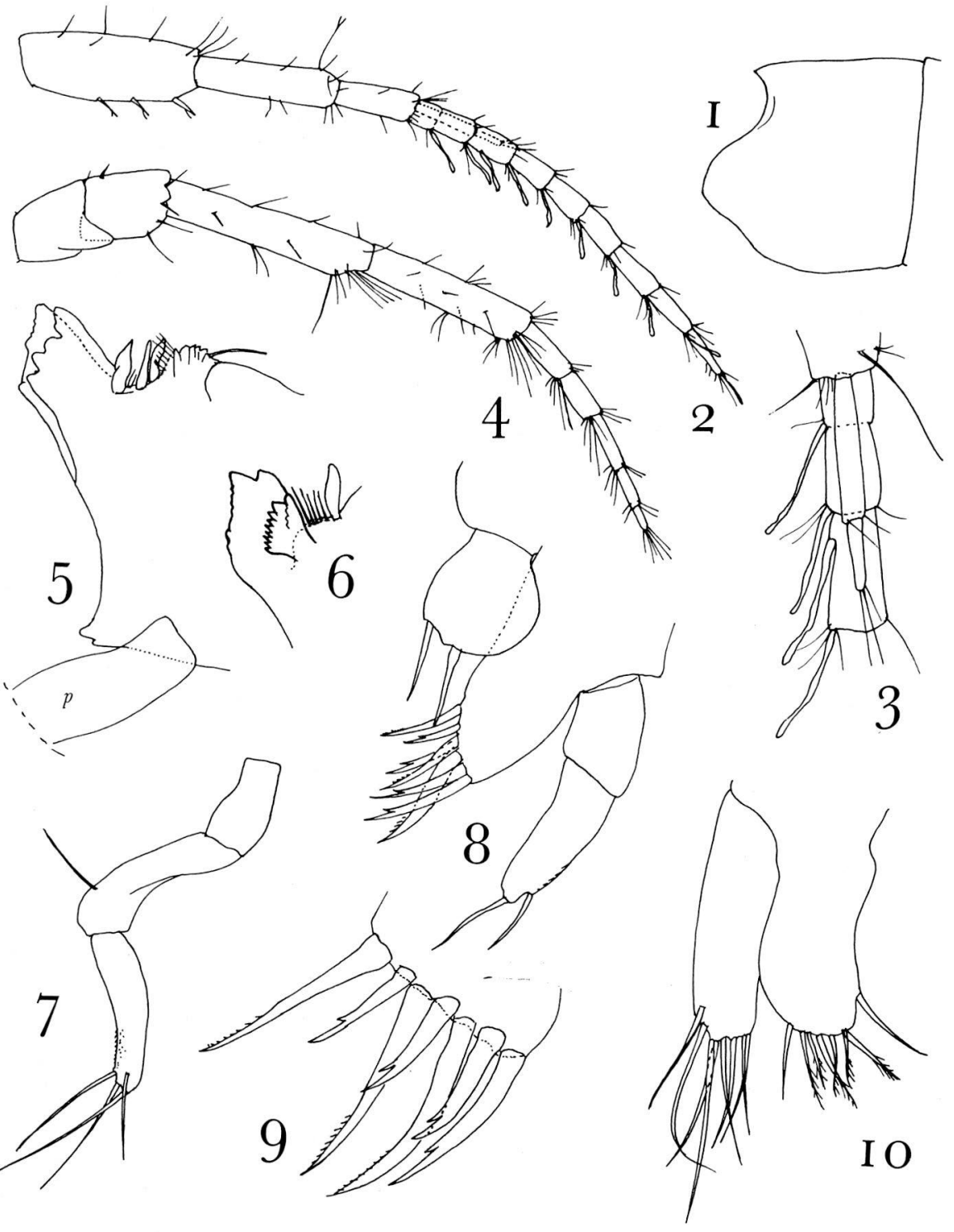

Figs. 1-10. Bogidiella martini n. sp., $\delta$ (holotype) et $q$ (paratype). 1, tête, $q$, de gauche (échelle a); 2 , antenne supérieure, $\delta$ (éch. b); 3, flagelle accessoire, $\hat{\partial}$ (éch. c); 4, antenne inférieure, $\hat{\delta}$ (éch. b); 5 , mandibule droite, $\hat{\delta}$ (éch. d) $(p=$ article basal du palpe); 6 , mandibule gauche, $\delta$ (éch. d); 7 , palpe mandibulaire, $\hat{\delta}$ (éch. e); 8 , maxille antérieure, $\delta$ (éch. f); 9 , armature de la lobe externe de la maxille antérieure, ô (éch. g); 10 , maxille postérieure, $\hat{\delta}$ (éch. f). 


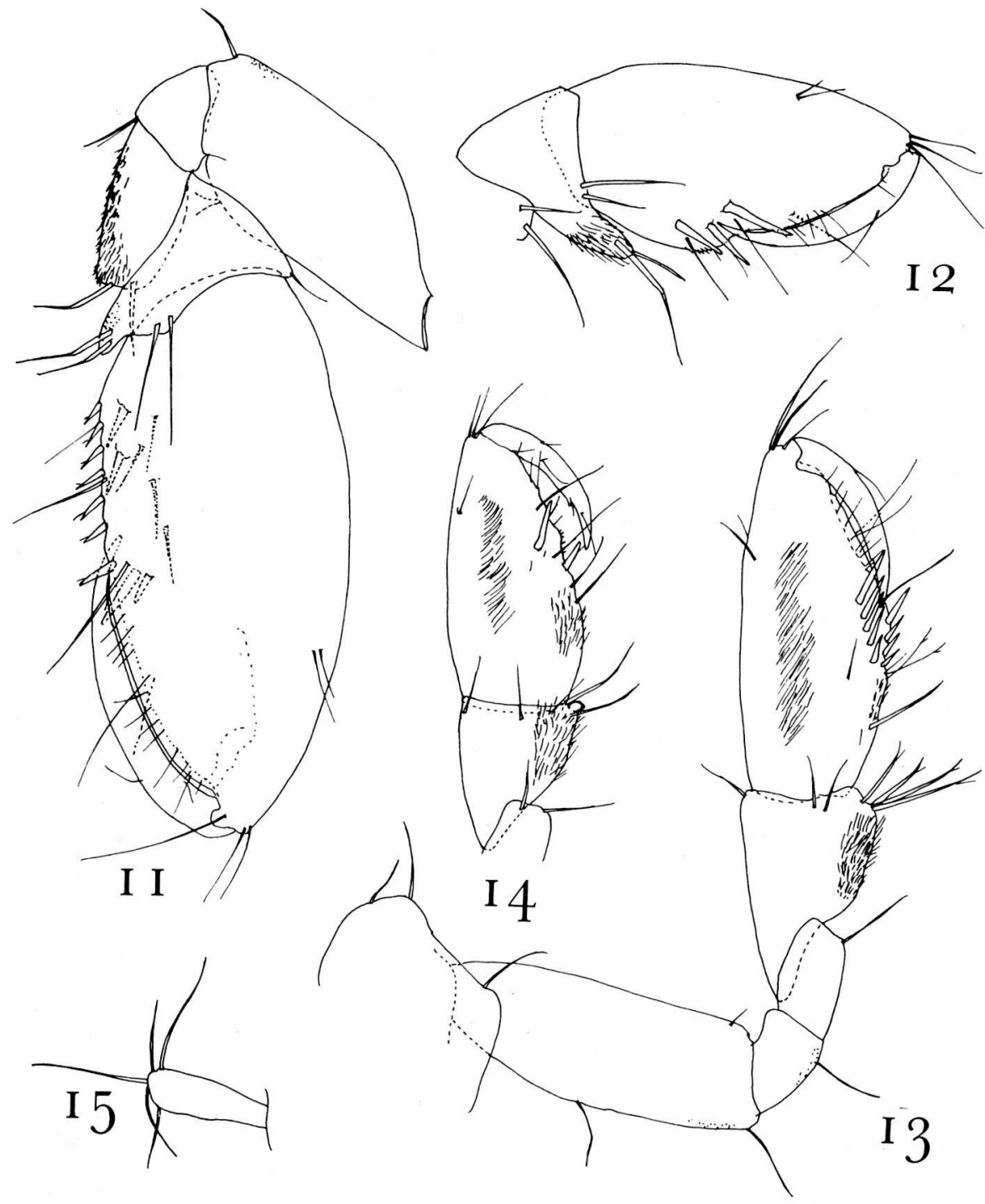

Figs. 11-15. Bogidiella martini n. sp., ô (holotype) et $Q$ (paratype). 11, gnathopode 1, ô (échelle a); 12 , articles distaux du gnathopode $1, q$ (éch. e); 13 , gnathopode 2 , ô (éch. a); 14 , articles distaux du gnathopode $2, q$ (éch. e); 15 , oostégite du gnathopode 2 , $q$ (éch. e). 

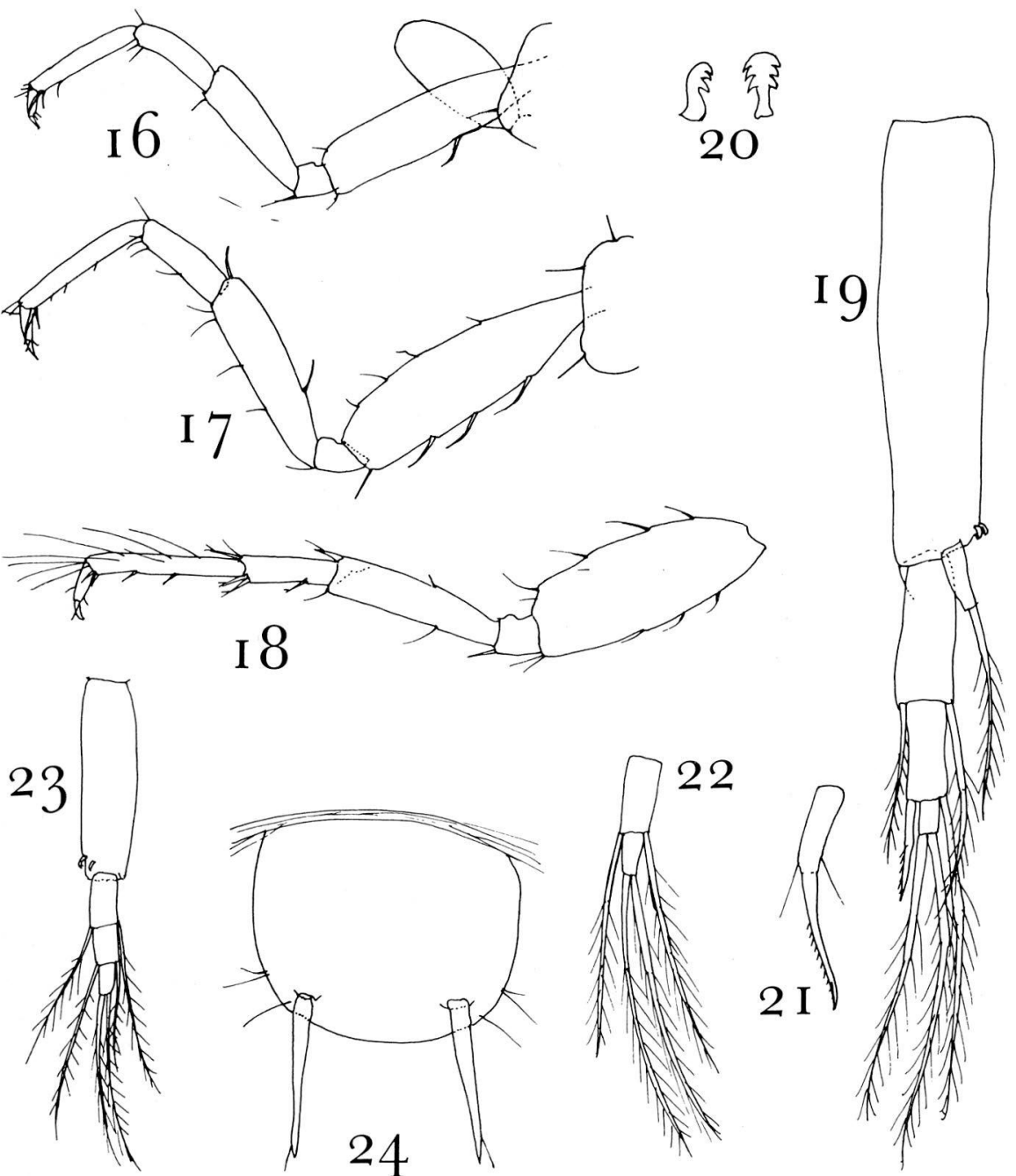

Figs. 16-24. Bogidiella martini n. sp., ô (holotype) et $q$ (paratype). 16, péréiopode 3, ô (échelle

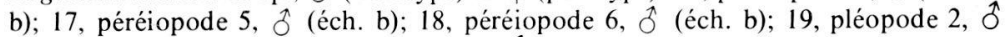
(éch. a); 20, rétinacles du 2ème pléopode, $\widehat{\partial}$ (croquis à main levée); 21 , élément modifié de l'exopodite du 2ème pléopode, ổ (éch. d): 22, articles distaux de l'exopodite du 3ème pléopode, $\hat{\sigma}$ (éch. a); 23, pléopode 2 , $q$ (éch. a); 24, telson, ổ (éch. h). 
médiane de soies longues et (caractère spécial pour cette espèce) une rangée de 4 épines robustes.

Gnathopode 1 beaucoup plus robuste que la gnathopode 2. Les deux gnathopodes montrent un dimorphisme sexuel prononcé dans leur propode.

Gnathopode 1, $\hat{\delta}$ (fig. 11): base avec 1 soie postérodistale et 2 soies antérodistales; les articles ischial, méral et carpal sans particularités. Propode régulièrement ovoïde; bord antérieur avec 2 courtes soies seulement; bord postérieur armé (et ceci est un des caractères distindifs de la présente espèce) avec une rangée de 6 épines marginales, pendant qu'on trouve 2 à 4 épines fortes à une distance faible du bord postérieur; l'angle palmaire est marqué de 3 épines (dont une très forte) et d'une soie; le bord palmaire n'est orné que de soies et de cils.

Chez la femellè, le propode (fig. 12) du gnathopode 1 est dilaté dans sa partie basale; l'angle palmaire est marqué de 3 épines très longues, d'une soie et d'une incurvation dans le bord palmaire; le bord postérieur est dépourvu d'épines.

Gnathopode 2, $\delta$ (fig. 13): article basal avec une petite soie antérodistale et 2 soies sur le bord postérieur. Propode allongé, 2,4 à 2,5 fois plus long que large; 2 rangées d'épines se trouvent compactement implantées sur la partie distale du bord postérieur et à l'angle palmaire (on y compte 9 épines, abstraction faite de la soie habituelle); un champ de cils se trouve sur "le plat" du propode.

Gnathopode 2, 9 (fig. 14): Le nombre de soies longues sur le carpe est inférieur à celui du mâle; l'angle postérodistal du carpe est pourvu d'un petit processus globuleux dont l'homologie reste incertaine. Propode en forme d'oeuf acuminé; le champ de cils sur "le plat" se retrouve chez la femelle comme che le mâle; le bord postérieur est dépourvu d'épines, mais orné de cils et de 2 soies; 2 épines et 1 soie sont implantées sur l'angle palmaire.

Oostégites présents aux gnathopodes 2 et péréiopodes 3,4 et 5 . Ils sont allongés et armés de 5 longues soies (fig. 15).

Branchies coxales elliptiques (fig. 16), présentes aux péréiopodes 4,5 et 6 .

Péréiopodes 3 et 4 très glabres (fig. 16) et similaires. Péréiopode 5 (fig. 17) avec quelques poils sur les articles basal et méral. Péréiopode 6 (fig. 18) avec un nombre de soies longues au bord postérieur du propode du mâle (absentes chez la femelle). Organes elliptiques absents. Les péréiopodes 7 sont amputés chez tous les individus examinés.

Pléopodes avec dimorphisme sexuel, qui se traduit dans la structure (uniramé chez la femelle, biramé chez le mâle) et dans la chétotaxie (avec un élément transformé au pléopode 2 du mâle). Les pléopodes mâles sont tous biramés (fig. 19): l'exopọdite est triarticulé; l'endopodite est uniarticulé, digitiforme, à taille considérable (entre $1 / 3$ et $1 / 2$ de la longueur du premier article de l'exopodite) et distalement pourvu d'une longue soie plumeuse. Le pédoncule porte 2 rétinacles, en forme d'ancre, avec 2 ou 3 crochets latéraux (fig. 20). Chez la femelle, il n'y pas de trace d'endopodite (fig. 23). L'élément latéral du 2ème article de l'exopodite du 2ème pléopode mâle n'est pas sétiforme, mais a été transformé en stylet légèrement courbe (fig. 21). Tous les autres éléments, chez les deux sexes, ont la forme d'une soie plumeuse. 

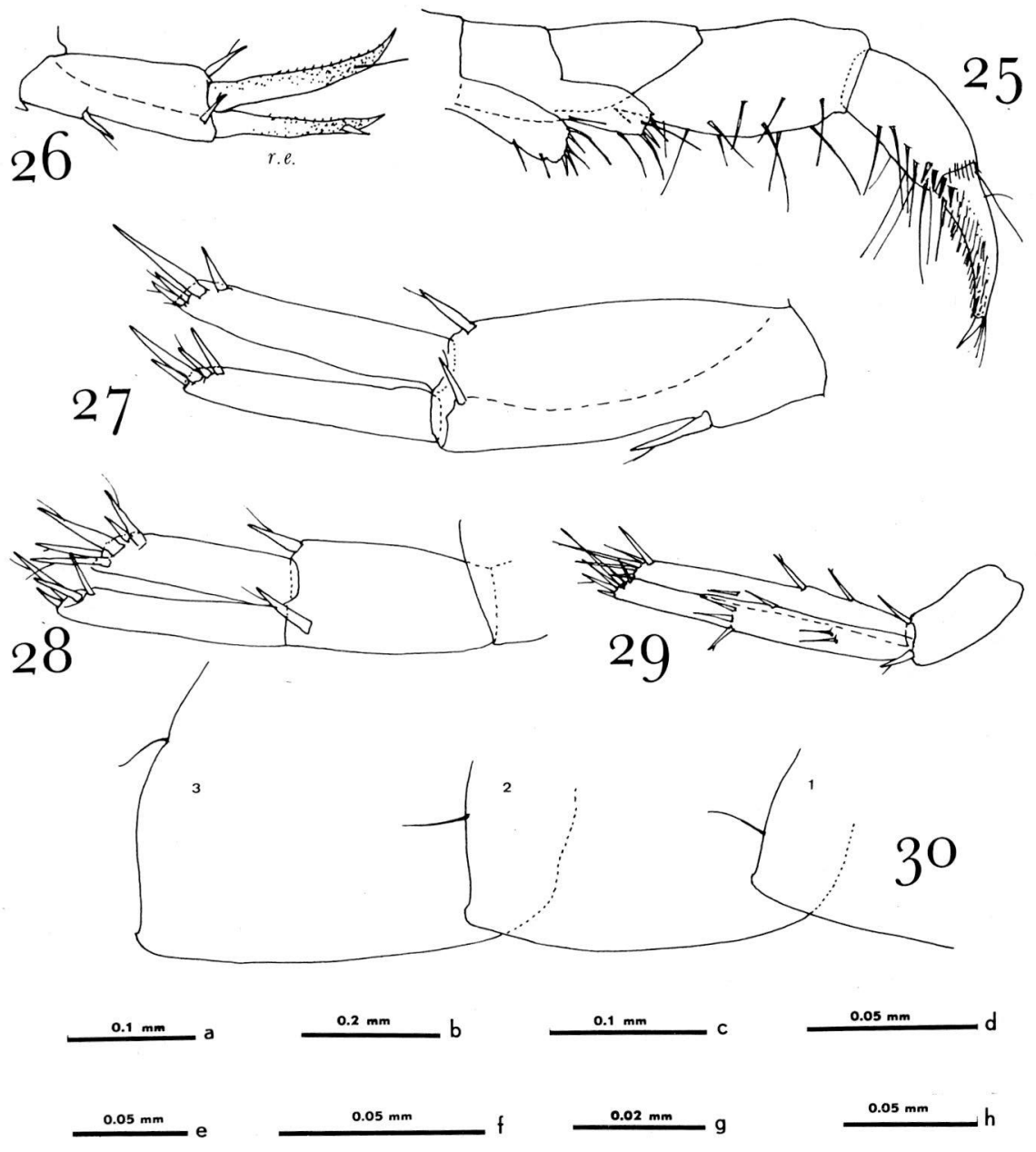

Figs. 25-30. Bogidiella martini n. sp., ô (holotype) et $९$ (paratype). 25, maxillipède, ô (échelle c); 26, uropode 1, ㅇ (éch. e) (r.e. = rame externe); 27, uopode 1, ô (éch. c); 28, uropode $2, \widehat{\partial}$ (éch. c); 29 , uropode 3 , ㅇ (éch. b); 30, plaques épimérales 1 à $3, \widehat{o}$ (éch. a).

Les plaques épimérales (fig. 30) possèdent chacune une soie sur leur bord postérieur; l'angle postéroventral est rectangulaire, marqué d'une légère échancrure.

L'uropode 1 montre un dimorphisme sexuel tres accentué: Chez le mâle (fig. 27), il est "normal", c.-à-d. avec deux rames subégales, droites, chacune armée, à son sommet, de 4 épines; le reste des rames est glabre. Chez la femelle (fig. 
26), les deux rames sont courbes et acuminées, la rame interne munie d'une soie subterminale, la rame externe d'une épine subterminale; les deux rames portant de nombreuses rugosités.

L'uropode 2 est identique dans les deux sexes, plus court que l'uropode 1, chaque rame droite, avec 4 ou 5 épines au sommet (fig. 28).

L'uropode 3 est sans dimorphisme sexuel (fig. 29), à rames allongées.

Le telson ne montre pas de dimorphisme non plus (fig. 24); il est un peu plus large que long, régulièrement arrondi, à marge distale convexe. Les deux épines distales sont plus courtes que le telson; près de leur base s'insèrent 3 petites soies.

Affinités. - Avec la clé dichotomique de Ruffo (1973) on peut suivre le sexe mâle de l'espèce nouvelle jusqu'aux couplets $18 / 19$, où il se retrouve avec $B$. longiflagellum S. Karaman, 1959, B. skopljensis (S. Karaman, 1933), B. helenae Mateus \& Maciel, 1967 (et B. tyrrhenica Schiecke, 1973, non encore comprise dans la clé de Ruffo). Contrairement à l'espèce nouvelle, ces espèces sont toutes dépourvues d'épines supplémentaires sur le bord postérieur du propode des gnathopodes 1 et $2(\hat{o})$. Les trois premières espèces (longiflagellum, skopljensis et helenae) ont un uropode 1 ( $q$.) normal, mais chez tyrrhenica cet appendice ressemble superficiellement à celui de notre espèce antillaise. Par contre, les pléopodes de tyrrhenica sont construits d'une manière tout à fait différente, et aussi dans le palpe mandibulaire (à 3ème article court, non-allongé) on trouve une nette distinction.

Le sexe femelle se laisse suivre dans la clé de Ruffo jusqu'au couplet 14. Là, notre expèce antillaise diffère de toutes les formes groupées sous le strophe 14a par l'absence d'organes elliptiques.

Si la présente espèce, avec un dimorphisme sexuel aussi prononcé que nous venons de décrire, avait été le premier cas dans le genre Bogidiella, j'aurais eu un doute quant à l'identité spécifique (et même générique) des deux sexes. Grâce à l'oeuvre de Mme. Coineau (1968), nous connaissons un cas similaire, quoique touchant parfois des appendices différents, à savoir l'espèce $B$. vandeli de la Sardaigne. Cependant, cette forme ne me semble pas étroitement parentée à l'espèce de St.-Martin, comme on le voit par son telson (à 4 épines), la structure de ses gnathopodes 1 et $2(q, \hat{o})$, par ses rames "normales" de l'uropode $1(q)$, etc.

Les seuls endroits, en dehors des récoltes sur l'île St.-Martin, d'où je connais du matériel antillais de Bogidiella, sont les îles de Barbuda et de Curaçao. De la première localité, on connaît une forme cavernicole de grande taille, $B$. bredini Shoemaker, 1959, dont le Dr. P. Wagenaar Hummelinck a pu ramasser deux exemplaires dans la localité-type, le Dark Cave, qu'il a aimablement mis à ma disposition pour l'étude morphologique. $B$. bredini n'est connue que de la localité-type. Elle se distingue de B. martini par la longueur de son corps (5-6 mm), par son telson (à 4 épines), par ses premiers uropodes femelles (non transformés), par son gnathopode 1 ( $q$ ) qui porte de nombreuses épines sur le bord postérieur du propode, par ses péréiopodes et griffes plus poilus, etc.

La deuxième forme, celle de Curaçao, n'est connue que de deux exemplaires 
BOGIDIELLA MARTINI N. SP. ET ZOOGEOGRAPHIE DES BOGIDIELLIDAE 111

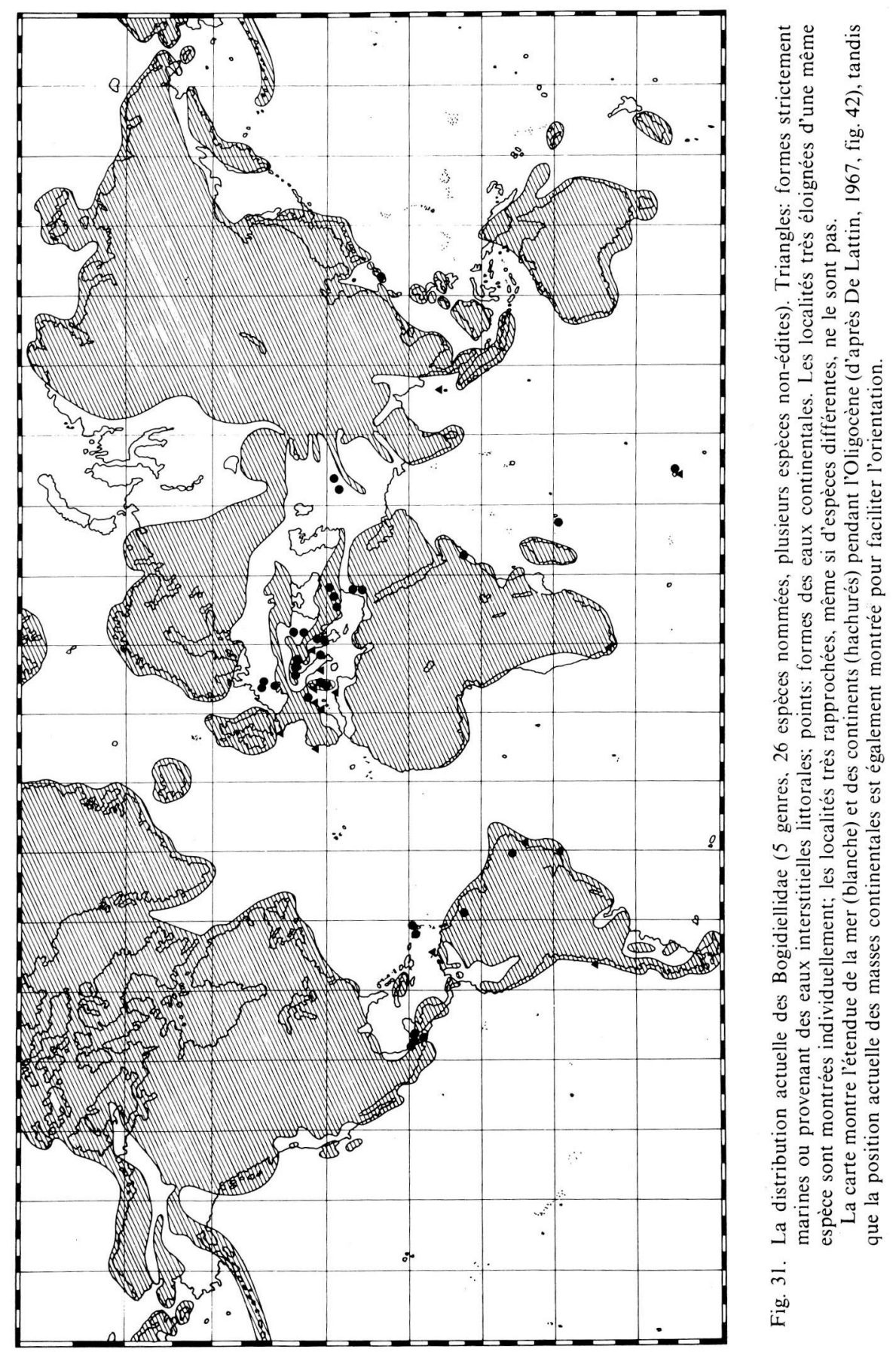


mutilés qui doivent rester inédits, provenant des sables grossiers littoraux de Playa Forti, Westpunt.

En conclusion, il n'y a pas de doute que la forme de l'île St.-Martin soit nouvelle; le nom spécifique proposé (martini, génitif de Martinus), fait allusion à la localité-type.

\section{LA ZOOGÉOGRAPHIE DES BOGIDIELLIDAE}

Les Bogidiellidae forment une famille de Crustacés Amphipodes dont les membres sont distribués dans la mer aussi bien que dans les eaux continentales. Cette particularité écologique n'est pas limitée aux Bogidielles, mais se retrouve pour d'autres groupes de Crustacés, p. ex. l'ordre des Thermosbaenacea, la famille des Hadziidae (Amphipoda) et la famille des Microparasellidae (Isopoda). La distribution actuelle des espèces de ces groupes semble correspondre très nettement avec l'extension oligocène de la Mer Téthys. Plusieurs auteurs, comme Ruffo (1957) ou Fryer (1965) ont signalé cette correspondance (tout au moins pour la partie de la Mer Téthys qui est devenue la Méditerranée actuelle) et ont supposé qu'une faune du littoral marin téthyen s'est échouée par suite d'oscillations géotectoniques pendant les périodes miocène et post-miocènes et s'est graduellement adaptée à un vie dans les eaux souterraines continentales.

Un autre groupe de Crustacés, le sous-ordre des Ingolfiellidea (Amphipoda), est également distribué dans tout le tracé marin - limnique. Mais là une correspondance nette entre les limites de la Mer Téthys et les aires de distribution des espèces n'est pas évidente. Stock (1977) suppose que, dans ce cas, il s'agit d'un groupe très ancien qui avait déjà atteint une grande partie de son aire de distribution avant la fragmentation du continent primordial de Pangée pendant l'ère Mésozoïque.

Les Bogidiellidae se conforment plutôt à ce dernier type, donc présentent une distribution résiduelle mésozoïque. La carte (fig. 31) montre la distribution actuelle des membres de cette famille. On reconnaît actuellement 5 genres, avec 26 espèces nommées, et plusieurs espèces non-édites. Les localités des formes du genre Bogidiella montrées sur la carte, sont basées sur les figures III et IV de Ruffo (1973), amplifiées avec mes propres données et avec les localités d'espèces des quatre autres genres considérés actuellement comme appartenant à la même famille, à savoir les genres Bollegidia (2 espèces), Pseudingolfiella (2 espèces), Spelaeogammarus (1 espèce) et Kergueniola (1 espèce).

La concentration d'espèces en Europe, surtout dans les pays méditerranéens, pourrait réfléter en partie les régions dans lesquelles les études sur les faunes interstitielle et souterraine ont été classiquement accomplies.

Malgré le fait que la plupart des espèces semble être distribuée dans des régions étant submergées par l'eau de mer pendant l'époque téthyenne (montrées en blanc sur la carte), il manque une corrélation nette entre le littoral de la Mer Téthys et les aires de distribution des Bogidiellidae. Points de correspondance entre le littoral téthyen et la distribution actuelle sont p. ex. la 
Mexique, les Petites Antilles, l'Italie, la Dalmatie et autres parties des Balkans, l'Afghanistan et la Somalie. D'autre part, la présence de membres des Bogidiellidés à l'intérieur du continent Sud-Américain, en Turquie, dans l'archipel des Kerguelen, au Japon.... ne s'explique pas facilement en termes d'une distribution résiduelle téthyenne, mais doit être considérée comme caractéristique des lignées confinées anciennes, sans doute d'âge mésozoïque.

L'existence de formes strictement marines et de nombreuses formes dans les eaux interstitielles littorales, indique que le processus d'invasion des eaux continentales n'est toujours pas terminé. Cette invasion s'est déroulée depuis l'ère mésozoïque, sans doute avec des optima pendant les régressions miocènes et post-miocènes, jusqu'à l'Holocène. Les espèces des Antilles, dont une fait l'objet de la note présente, sont originaires de l'époque des régressions tertiaires (les Petites Antilles étant submergées pendant le Tertiaire inférieur).

\section{RÉSUMÉ}

Les deux sexes d'une espèce nouvelle de Bogidiella, B. martini, sont décrits. Cette espèce, à dimorphisme sexuel très prononcé, a été découverte dans deux puits à St.-Martin (partie française), une des îles des Petites Antilles. Un autre membre de Bogidiella est signalé de l'île de Curaçao, mais les exemplaires sont trop mutilés pour une description satisfaisante.

Les Bogidiellidae ( 5 genres, 26 espèces nommées, plusieurs espèces non-édites) sont réparties dans la mer aussi bien que dans les eaux continentales. La famille présente une vaste distribution, dépassant l'étendue de l'ancienne Mer Téthys. Probablement il s'agit d'un groupe très ancien qui avait déjà atteint une grande partie de son aire de distribution avant la fragmentation du continent primordial de Pangée pendant l'ère Mésozoïque.

\section{BIBLIOGRAPHIE}

COINEAU, N. (1968). Contribution à l'étude de la faune interstitielle - Isopodes et Amphipodes. Mém. Mus. natn. Hist. nat., (n.S.) (A) Zool., 55 (3): 145-216.

FRYER, G. (1965). Studies on the functional morphology and feeding mechanism of Monodella argentarii Stella (Crustacea: Thermosbaenacea). Trans. roy. Soc. Edinburgh, 66 (4): 49-90.

LATTIN, G. DE (1967). Grundriss der Zoogeographie: 1-602. (Gustav Fischer, Jena).

RUFFO, S. (1957). Le attuali conoscenze sulla fauna cavernicola della regione Pugliese. Mem. Biogeogr. Adriat., 3: 1-143.

- (1973). Contributo alla revisione del genere Bogidiella Hertzog (Crustacea Amphipoda, Gammaridae). Boll. Ist. Entom. Bologna, 31: 49-77.

SCHIECKE, U. (1973). Ein Beitrag zur Kenntnis der Systematik, Biologie und Autökologie mariner Peracarida (Amphipoda, Isopoda, Tanaidacea) des Golfes von Neapel: 1-408, 91 pls. (Inaug. Diss. Christian-Albrechts-Univ., Kiel).

STOCK, J. H. (1977). Taxonomy and zoogeography of the crustacean suborder Ingolfiellidea, with emphasis on the West Indian taxa. Stud. Fauna Curaçao, 55: 131-146. 